\title{
Distractibility in Daily Life Is Reflected in the Structure and Function of Human Parietal Cortex
}

\author{
Ryota Kanai, ${ }^{1}$ Mia Yuan Dong, ${ }^{2}$ Bahador Bahrami, ${ }^{1,3,4,5}$ and Geraint Rees ${ }^{1,3}$ \\ ${ }^{1}$ Institute of Cognitive Neuroscience and ${ }^{2}$ Department of Psychology, University College London, London WC1N 3AR, United Kingdom, ${ }^{3}$ Wellcome Trust \\ Centre for Neuroimaging, Institute of Neurology, University College London, London WC1N 3BG, United Kingdom, ${ }^{4}$ Interacting Minds Project, Institute of \\ Anthropology, Archaeology, and Linguistics, Aarhus University, and ${ }^{5}$ Centre of Functionally Integrative Neuroscience, Aarhus University Hospital, 8000 \\ Aarhus C, Denmark
}

We all appreciate that some of our friends and colleagues are more distractible than others. This variability can be captured by pencil and paper questionnaires in which individuals report such cognitive failures in their everyday life. Surprisingly, these self-report measures have high heritability, leading to the hypothesis that distractibility might have a basis in brain structure. In a large sample of healthy adults, we demonstrated that a simple self-report measure of everyday distractibility accurately predicted gray matter volume in a remarkably focal region of left superior parietal cortex. This region must play a causal role in reducing distractibility, because we found that disrupting its function with transcranial magnetic stimulation increased susceptibility to distraction. Finally, we showed that the self-report measure of distractibility reliably predicted our laboratory-based measure of attentional capture. Our findings distinguish a critical mechanism in the human brain causally involved in avoiding distractibility, which, importantly, bridges self-report judgments of cognitive failures in everyday life and a commonly used laboratory measure of distractibility to the structure of the human brain.

\section{Introduction}

The ability to select goal-relevant information while ignoring irrelevant distraction is essential for survival. In everyday life, selection of incoming information is accomplished via two functionally distinct but complementary attention mechanisms (Corbetta and Shulman, 2002). A voluntary top-down control mechanism directs attention to objects and events relevant to current goals (Desimone and Duncan, 1995; Kastner and Ungerleider, 2000); but attention can be also captured automatically in a bottom-up fashion by salient stimuli in the environment (Yantis and Jonides, 1984, 1990; Theeuwes, 1991; Itti and Koch, 2001). If top-down control is insufficient, then the bottom-up mechanism dominates, leading to distractibility and difficulty in maintaining attention on the relevant task. Distraction can impair productivity and increase the risk of accidents (Larson and Merritt, 1991; Wallace and Vodanovich, 2003).

Across individuals, there is a large variability in the ability to maintain attention in the presence of distractors (Davies et al., 1984). The Cognitive Failures Questionnaire (CFQ) (Broadbent et al., 1982) consists of 25 items that assess everyday absentmindedness and failures of attention, and thus indexes this variation in

Received Nov. 8, 2010; revised Feb. 27, 2011; accepted March 3, 2011.

Author contributions: R.K., M.Y.D., B.B., and G.R. designed research; R.K., M.Y.D., and B.B. performed research; R.K., M.Y.D., and B.B. analyzed data; R.K., M.Y.D., B.B., and G.R. wrote the paper.

This study was funded by the Wellcome Trust (G.R.), the Human Frontier Science Program (R.K.), the European Union MindBridge project (B.B., G.R.), and the MINDLab UNIK initiative at Aarhus University funded by the Danish Ministry of Science, Technology, and Innovation (B.B.).

Correspondence should be addressed to Dr. Ryota Kanai, UCL Institute of Cognitive Neuroscience, 17 Queen Square, London WC1N 3AR, United Kingdom. E-mail: r.kanai@ucl.ac.uk.

DOI:10.1523/JNEUROSCI.5864-10.2011

Copyright $\odot 2011$ the authors $\quad 0270-6474 / 11 / 316620-07 \$ 15.00 / 0$ distractibility. The CFQ is a simple self-report assessment; nonetheless, previous studies have established its reliability and validity in quantifying the distractibility of individuals. CFQ scores are stable over a long period of time, with a marked test-retest consistency even after 16 months (Broadbent et al., 1982), and scores from self-report highly correlate with those derived from ratings by their spouses. Moreover, CFQ scores predict attention performance in laboratory settings (Tipper and Baylis, 1987; Forster and Lavie, 2007). Individuals with high CFQ scores are slow at responding to a target in the presence of concurrent distracting stimuli, while in the absence of distractors, their performance is comparable to individuals with low CFQ scores (Tipper and Baylis, 1987). CFQ scores also predict performance in divided attention tasks (Martin and Jones, 1983). These findings indicate that CFQ scores provide consistent and reliable estimates of an individual's ability to suppress task-irrelevant distraction.

Surprisingly, susceptibility to distraction is highly heritable. A study of $>3000$ Dutch adolescent twins and their biological parents assessed heritable contributions to CFQ by comparing the similarity of CFQ scores within pairs of monozygotic twins to that within pairs of dizygotic twins (Boomsma, 1998). The results revealed that as much as $50 \%$ of interindividual variability in CFQ scores can be attributed to genetic factors. This degree of heritability suggests that variability in CFQ scores may be mediated by genetic influences on the brain, which may be expressed via variability in brain structure.

We therefore hypothesized that interindividual variability in distractibility would be associated with differences in brain structure involved in control of selective attention. To test this hypothesis, we measured both CFQ scores and magnetic resonance imaging (MRI) brain scans in 145 healthy adult individuals. We tested whether the 
distractibility component of the CFQ (derived from the standardized loadings reported in previous studies-see Materials and Methods for full details) predicted brain structure using optimized voxel-based morphometry (VBM). We focused our analyses on the frontal and parietal lobes, as they are considered critical for attentional control (e.g., Corbetta and Shulman, 2002).

\section{Materials and Methods}

Experiment 1: voxel-based morphometry of everyday distractibility Participants. For the VBM experiment, a total of 145 healthy volunteers with normal or corrected-to-normal vision (aged 18-32, mean $23.6 \pm$ 4.76 SD, 95 female) were recruited from the University College London subject pool. The experiments were approved by the local ethics committee.

Assessment of daily distractibility. All participants were asked to fill out the CFQ, which was used to assess distractibility in daily life (Broadbent et al., 1982). The CFQ requires respondents to rate the frequency with which they experience 25 common cognitive failures in perception, memory, and motor function on a scale of 0 to 4 , with 0 indicating never and 4 meaning very often, e.g., "How often do you fail to see what you want in a supermarket (although it's there)?"

While the CFQ is generally considered to reflect an individual's distractibility, the questionnaire also encompasses a broad range of common errors in everyday situations. Previous factor-analysis studies identified that the CFQ consists of four principal factors, i.e., distractibility, memory, blunders, and names (Wallace et al., 2002; Wallace, 2004). To focus on the distractibility component, we extracted the distractibility subscale using the standardized loadings derived from the previous factor analysis (Wallace et al., 2002).

MRI data acquisition. MR images were acquired on a $1.5 \mathrm{~T}$ Siemens Sonata MRI scanner (Siemens Medical). High-resolution anatomical images were acquired using a T1-weighted three-dimensional modified driven equilibrium Fourier transform sequence (repetition time $=12.24$ $\mathrm{ms}$; echo time $=3.56 \mathrm{~ms}$; field of view $=256 \times 256 \mathrm{~mm}$; voxel size $=1 \times$ $1 \times 1 \mathrm{~mm})$.

$V B M$ preprocessing and analysis. T1-weighted MR images were first segmented for gray matter (GM) and white matter using the segmentation tools in Statistical Parametric Mapping 8 (SPM8, http://www.fil.ion. ucl.ac.uk/spm). Subsequently, we performed diffeomorphic anatomical registration through exponentiated lie algebra (DARTEL) in SPM8 for intersubject registration of the GM images (Ashburner, 2007). To ensure that regional gray matter volume is maintained after the registration, the registered images were modulated by the Jacobian determinant of the flow fields computed by DARTEL. The registered images were smoothed with a Gaussian kernel of $8 \mathrm{~mm}$ full-width at half-maximum and were then transformed to Montreal Neurological Institute (MNI) stereotactic space using affine and nonlinear spatial normalization implemented in SPM8.

A multiple regression analysis was performed on the smoothed gray matter images in SPM8 to determine regions in which gray matter density showed a positive correlation with everyday distractibility. We constrained our search volume to frontal and parietal lobes using a standard atlas to construct a mask for those lobes together (MNI structural atlas) (Collins et al., 1995; Mazziotta et al., 2001). The total gray matter volume of individual brain was included in the design matrix to regress out the general size difference across the participants. We used a threshold of $p<$ 0.05 , corrected for multiple comparisons across the frontoparietal mask volume using the familywise error rate (FWE).

\section{Experiment 2: attentional capture and transcranial magnetic stimulation}

Participants. Eighteen healthy participants with normal or corrected-tonormal vision (aged 19-35, mean $=24.3 \pm 4.9 \mathrm{SD}, 14$ female) were recruited. Since the purpose of this experiment was to observe effects of transcranial magnetic stimulation (TMS) on attentional capture, we selected participants who showed some degree of attentional capture to avoid floor effects in case TMS turned out to reduce attentional capture. Data from two participants were discarded on this criterion because they did not show any effects of attentional capture in the baseline condition (i.e., $<0 \mathrm{~ms}$ ). Another one participant was rejected because he performed the task very poorly (accuracy was $57.5 \%$, not significantly above chance in the incongruent condition). All of the remaining 15 participants (age 19-35, mean $24.1 \pm 5.2 \mathrm{SD}, 3$ male) were right handed. In both experiments, we obtained written informed consent from all participants before the TMS session. The local ethics committee at University College London approved the experiments.

Stimuli and procedure. The stimuli were a visual search array presented at $57 \mathrm{~cm}$ from the observer's eyes, against a black background on a 19 inch monitor using Cogent Toolbox (http://www.vislab.ucl.ac.uk/cogent.php) running under MATLAB (The MathWorks).

The experimental display (see Fig. $2 A, B$ ) consisted of five shapes that were equally spaced in a circular arrangement, presented at $9^{\circ}$ eccentricity from a central fixation point. Shapes consisted of four diamonds (diameter: $2.25^{\circ}$ ) serving as distractors and one target circle (diameter: $2.25^{\circ}$ ). In the center of each shape was a white line segment (length: $0.68^{\circ}$; thickness: $0.1^{\circ}$ ) randomly assigned to be either horizontal or vertical. Participants were instructed to respond to the orientation of the line segment inside the target circle as quickly and as accurately as possible by pressing " $\mathrm{B}$ " to indicate horizontal and " $\mathrm{N}$ " for vertical on a keyboard, using their left and right index fingers, respectively. In $50 \%$ of trials, the target circle was red and all diamonds were green (see Fig. 2 A). In these trials, task-irrelevant color singleton (red) was congruent with the taskrelevant shape singleton (circle). In the remaining trials, one of the diamonds (but not the circle) was red, meaning that task-relevant shape and color singletons were incongruent (see Fig. $2 \mathrm{~B}$ ). Congruent and incongruent trials were pseudorandomly interleaved.

Each experimental trial began with the presentation of a fixation point for duration between 500 and $1000 \mathrm{~ms}$ chosen from a uniform random distribution, followed by the presentation of the visual search array. The search array was then presented on the screen for $500 \mathrm{~ms}$, followed by a response period of $1600 \mathrm{~ms}$. Participants were asked to maintain eye fixation throughout the experiment.

Before the main experiment, participants were given a practice block (20 trials) to ensure that they understood the task and instructions. Feedback regarding accuracy was given in the practice block, but not in the main blocks. Participant completed two runs ( 12 blocks in each run, 20 trails in each block) before receiving TMS and another two runs after TMS, resulting in a total of 960 trials. The first run was treated as a practice session. The two stimulation sites [left superior parietal lobe (SPL) and vertex] were tested on separate days and the order of the sites was counterbalanced across participants.

Analysis. The size of attentional capture was computed as the difference between the mean reaction time (RT) in the incongruent condition minus the mean RT in the congruent condition. In computing the mean RTs, on average, $9.6 \%$ of trials were excluded from analysis due to incorrect responses and $<0.1 \%$ due to excessively slow response ( $>1500 \mathrm{~ms}$ ).

The items in the search display were arranged such that two items were in the left visual field (LVF) and two items in the right visual field (RVF) (see Fig. 2A,B). One item was along the vertical meridian above the fixation point. In the analysis where we report the performance in LVF and RVF separately, those four target positions were used and the trials in which the target appeared at the top position were omitted to avoid ambiguity.

TMS protocol. We delivered the standard continuous theta-burst stimulation (cTBS) protocol, i.e., three pulses at $50 \mathrm{~Hz}$ repeated at $200 \mathrm{~ms}$ intervals for $40 \mathrm{~s}$. The TMS pulses were delivered by a Magstim Rapid 2 at $40 \%$ of stimulator output using a figure-of-eight coil. This protocol is known to depress cortical excitability at the stimulated region for up to $60 \mathrm{~min}$ (Huang et al., 2005). The position of the left SPL was obtained by converting the coordinates obtained from the VBM analysis (above) for individual participants using FSL software (FMRIB, Oxford, UK). The target sites determined for individual participants were used to guide TMS coil position using a frameless stereotaxic system (Brainsight, Rogue Research). 
A

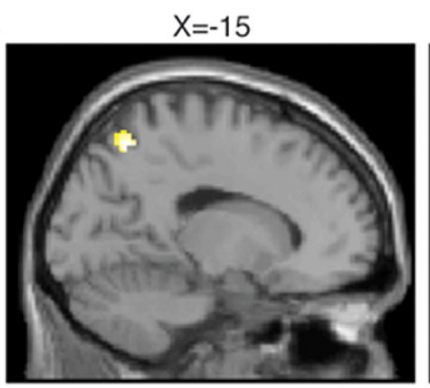

B

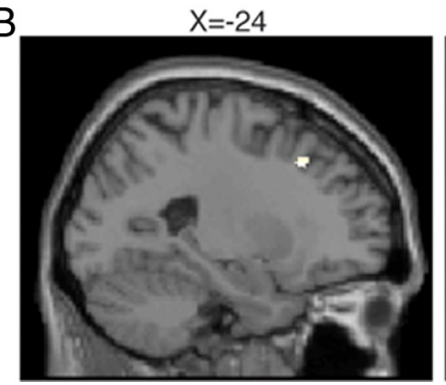

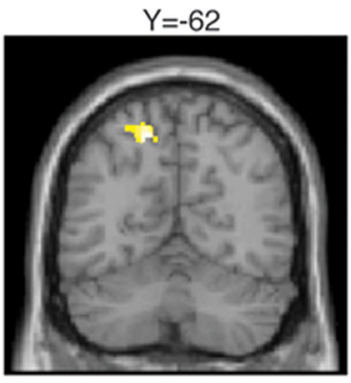
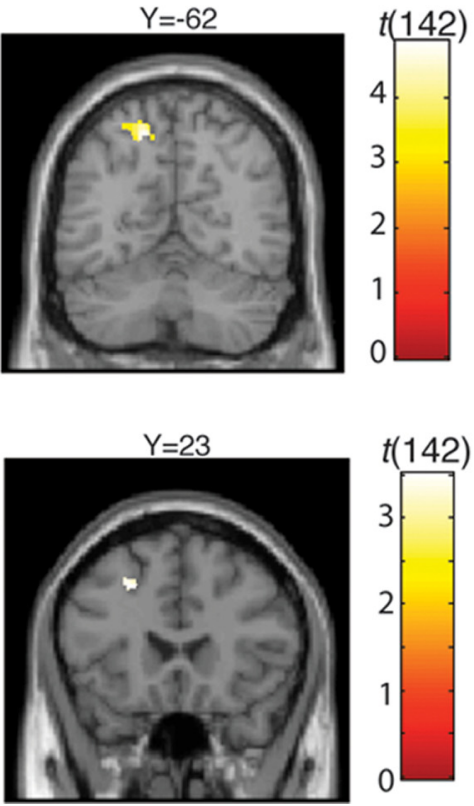

C

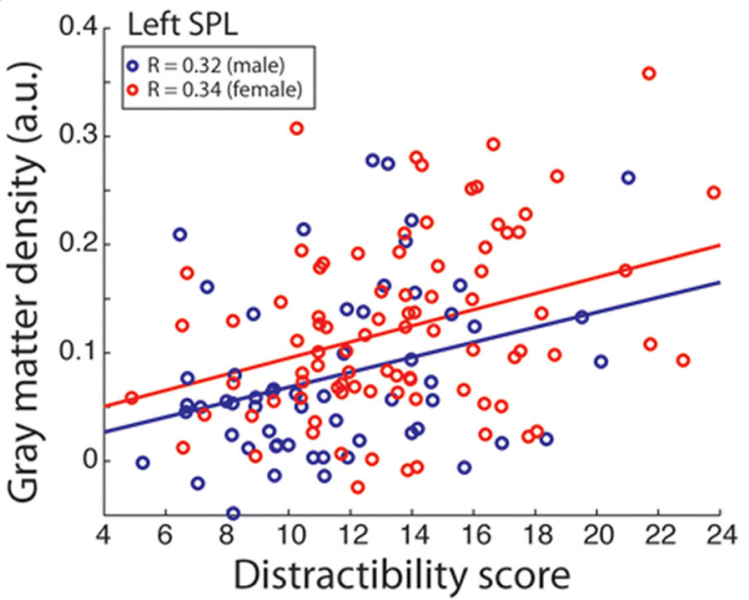

Figure 1. $A$, Cluster in the left superior parietal lobe showing positive correlation with distractibility in everyday life. The threshold is set to $p<0.001$ uncorrected for display purposes only. This cluster survived correction for multiple comparisons $\left(p_{\mathrm{FWE}}<0.05\right.$; see Materials and Methods for full details). $\boldsymbol{B}$, Cluster in the left prefrontal cortex showing negative correlation. However, this area did not survive correction for frontoparietal mask volume $\left(p_{\mathrm{FWF}}=0.755\right)$. $C$, The gray matter density of the peak voxel of the left SPL is plotted against distractibility score separately for male (shown in blue) and female (shown in red). Regression lines are also fitted to male and female participants separately.

Experiment 3: attentional capture and CFQ

Participants. Data were collected from a total of 28 healthy participants with normal or corrected-to-normal vision (aged 18-35, mean $=23.5 \pm$ $4.8 \mathrm{SD}, 24$ female). Attentional capture data from the 15 participants completing the pre-TMS baseline condition on the first day in the TMS experiment (experiment 2) were combined with new data from additional 13 participants.

Stimuli and procedure. The stimuli and procedure of the experiment were identical to the TMS experiment except that the new participants completed only the two blocks, which corresponded to the pre-TMS condition (12 blocks in each run, 20 trails in each block). As in the TMS experiment, we treated the first block as a practice run and used the data from the second run for estimating the size of attentional capture. When we estimated individuals' attentional capture size from the data obtained in experiment 2, we used the data from the pre-TMS condition on the first day. Therefore, the exposures to the task at the time when the degree of attentional capture was measured were identical between the two groups. All participants filled out the CFQ (Broadbent et al., 1982).

\section{Results}

Voxel-based morphometry and distractibility

Our VBM analysis revealed that interindividual variability in the gray matter volume of the left SPL positively correlated with distractibility indexed by the CFQ scores (Fig. 1A) (MNI coordinate: $x=-15, y=-61, z=54, r=0.380, t_{(142)}=4.88, p=0.015$ FWE corrected for parietal and prefrontal cortical volume examined; see Materials and Methods). No other brain region reached statistical significance for a positive correlation between distractibility and gray matter volume $(p<0.05$, FWE corrected for either whole brain or parietal and prefrontal cortex). A weak negative correlation between gray matter volume and distractibility was found in the left mid prefrontal cortex (Fig. 1B) $(x=-24, y=23$, $z=40, r=-0.283, t_{(142)}=3.51, p<0.001$ uncorrected). However, this did not reach statistical significance after correction for multiple comparisons ( $p=0.755$, FWE corrected).

As has been reported in previous studies (e.g., Boomsma, 1998), we found a significant gender difference in distractibility scores. Specifically, female participants had higher distractibility scores than male participants [13.5 \pm 3.7 (SD) vs $11.5 \pm 3.6(\mathrm{SD})$; $t$ test, $\left.t_{(143)}=3.31, p<0.01\right]$. This raised the concern that the observed gray matter correlates might be driven primarily by gender differences in brain structure. To examine this possibility, we computed the correlation between the left SPL and distractibility for male and female groups separately (Fig. 1C). We found comparable and statistically significant correlations between the gray matter density of the left SPL and distractibility scores for both male and female groups (male, $t_{(52)}=2.52, r=0.320, p=$ 0.014 ; female, $t_{(85)}=3.36, r=0.342, p=0.001$ ). These withingender correlations show that the correlation between the structure of the left SPL and everyday distractibility that we observed was not driven by gender differences.

The positive correlation between gray matter density of the left SPL and the distractibility score has at least two possible functional interpretations. First, increased gray matter density of the left SPL might make people more sensitive to bottom-up saliency, thereby making it difficult to ignore task-irrelevant stimuli. We will refer to this as the bottom-up saliency hypothesis. The second possibility, which we will refer to as the top-down control hypothesis, is that the left SPL exerts top-down control to suppress distraction from bottom-up saliency. This implies - paradoxically-that a lower gray matter density in SPL is associated with greater effectiveness at suppressing task-irrelevant stimuli (see also Discussion). To distinguish these two hypotheses, we next examined how TMS over the left SPL region influenced individual distractibility in a new group of 18 healthy adult participants (see Materials and Methods for full details).

\section{Effects of TMS over left SPL on attentional capture}

To quantify distractibility before and after TMS, we used an attentional capture paradigm (Theeuwes, 1991, 1992, 1994; de Fockert et al., 2004) in which a salient yet task-irrelevant stimulus interferes with goal-relevant behavior. In the attentional capture 

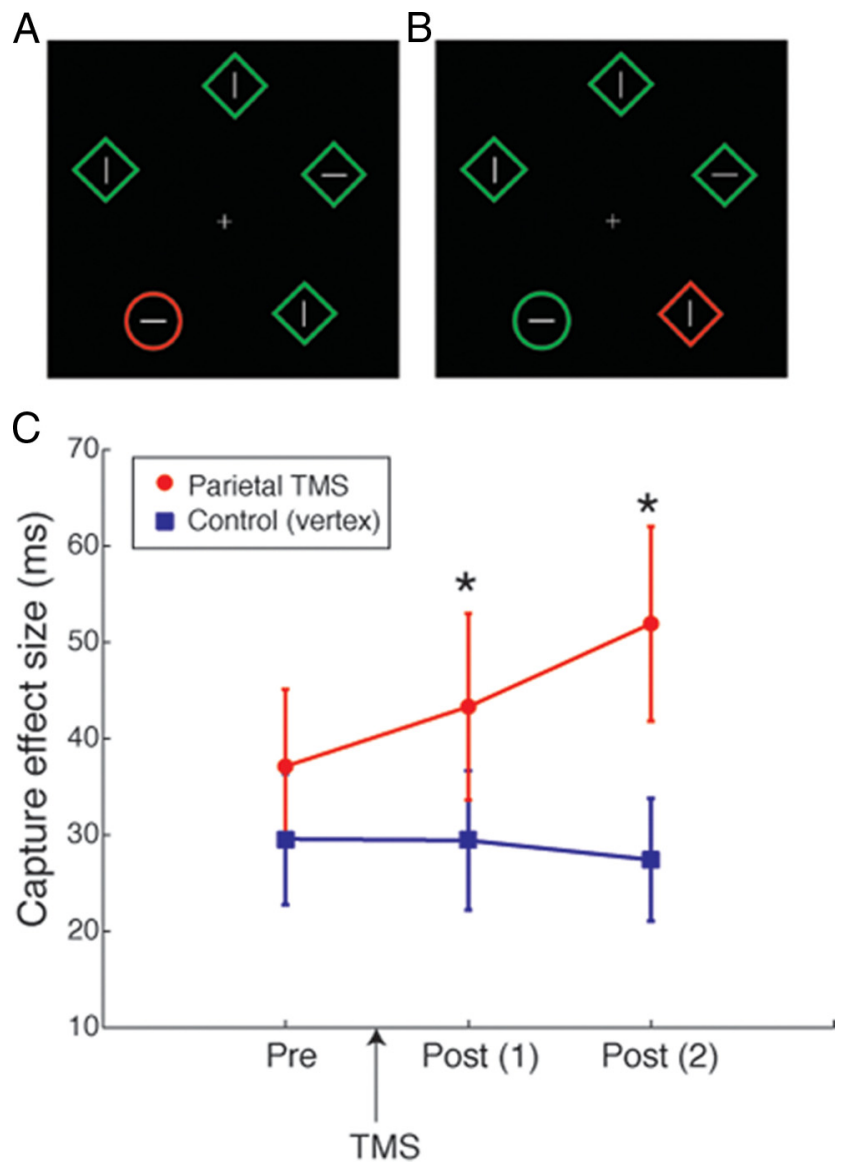

Figure 2. $\boldsymbol{A}, \boldsymbol{B}$, Example stimuli for congruent $(\boldsymbol{A})$ and incongruent $(\boldsymbol{B})$ trials in the TMS experiment (see Materials and Methods for full details). Participants reported the orientation of the line inside the target circle. The red color singletons were irrelevant to the task, but could be congruent $(\boldsymbol{A})$ or incongruent $(\boldsymbol{B})$ with the target. The two types of trials were intermixed within the same block. $\boldsymbol{C}$, The capture effects before and after theta-burst TMS are plotted per TMS site. The red circles indicate the parietal TMS condition and the blue squares the control condition. Error bars indicate 1 SEM across 15 participants. The asterisks indicate a significant difference between the conditions $(p<0.05)$.

paradigm, the delay in a visual search task by the presence of an irrelevant yet salient stimulus was taken as the measure of distraction (see Materials and Methods for full details).

Our two hypotheses regarding the role of the left SPL made different predictions for this TMS experiment: The bottom-up saliency hypothesis predicted that suppression of the left SPL with TMS would decrease the amount of attentional capture. In contrast, the top-down control hypothesis predicted that disrupting the function of this area with TMS would lead to increased attentional capture.

Figure $2 C$ shows the results of the TMS experiment. We measured attentional capture before TMS and after application of TMS to either the vertex (control) or left SPL. Before TMS, attentional capture did not differ for vertex or SPL conditions (for pre-TMS, $t_{(14)}=0.78 ; p=0.44$; paired $t$ test comparing vertex and SPL conditions). After application of the TMS to these sites, significant differences in attentional capture were found. Attentional capture was significantly larger when TMS was applied to SPL compared to vertex (for the first post-TMS session, $t_{(14)}=$ $2.289 ; p=0.038$; for the second post-TMS session, $t_{(14)}=2.286$; $p=0.038)$. This significant increase in attentional capture supports the hypothesis that the left SPL is involved in top-down

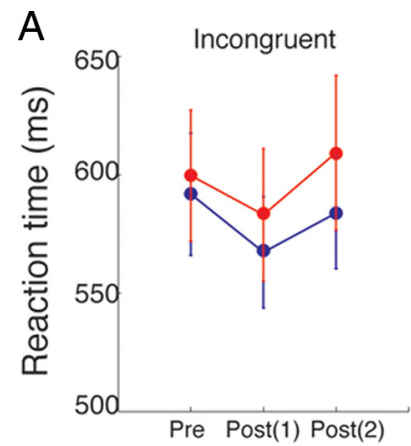

B
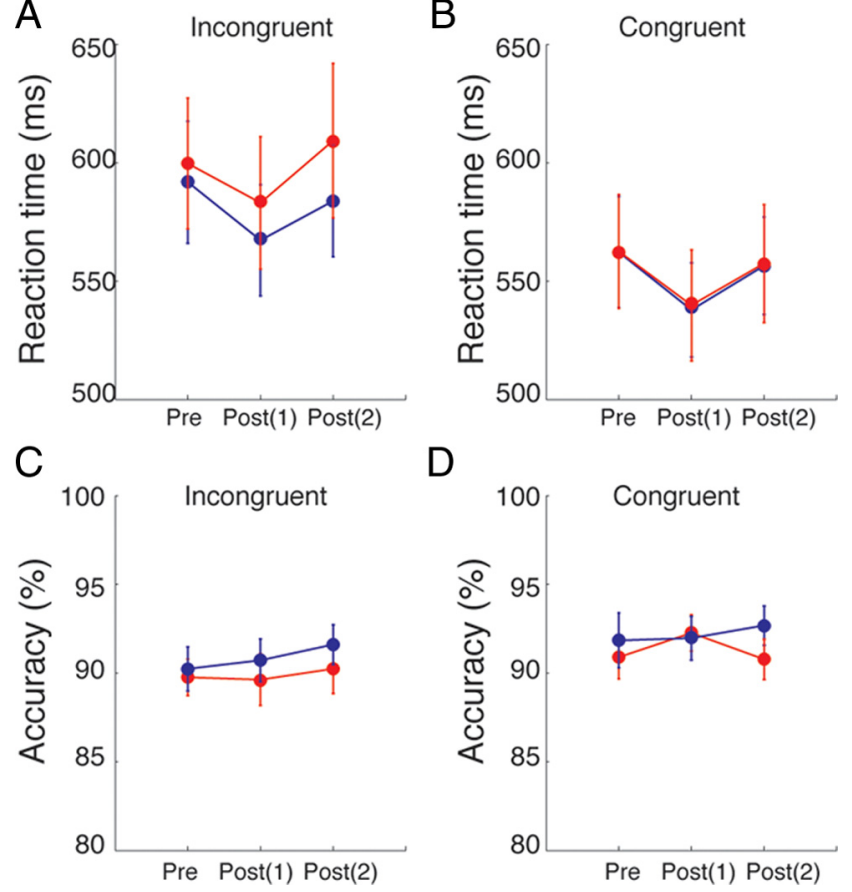

Figure 3. Reaction time and accuracy per condition. $\boldsymbol{A}$, The mean RT for incongruent trials. $\boldsymbol{B}$, The mean RT for congruent trials. $\boldsymbol{C}$, The mean accuracy for incongruent trials. $\boldsymbol{D}$, The mean accuracy for congruent trials. In all panels, red circles indicate the condition with TMS over the left SPL and blue circles the control condition with TMS over the vertex. Error bars indicate 1SEM across 15 participants.

control of attention by maintaining attention to task-relevant stimuli while suppressing salient distractors.

To understand whether the increase in attentional capture was driven by performance changes in incongruent trials or both congruent and incongruent trials, we further analyzed the RTs and accuracy in congruent and incongruent trials separately. The figure shows that TMS over the left SPL did not affect the RTs in congruent trials (Fig. 3B), whereas it selectively increased RTs for incongruent trials (Fig. 3A). While the differences in RTs in the incongruent trials were only marginally significant (two postTMS sessions combined: $t_{(14)}=1.34, p=0.09$ ), this analysis suggests that the significant effect observed in attentional capture was driven by the change in incongruent trials. A further analysis on accuracy did not show any difference between the left SPL and control conditions in either incongruent (Fig. 3C) or congruent (Fig. 3D) trials. The selective effect on incongruent trials further provide support for the notion that the function of the left SPL is to avoid distraction from task-irrelevant, salient stimuli.

Finally, we examined whether the effect of TMS over left SPL was specific to the trials in which the target appeared contralateral to the TMS site (i.e., RVF) or bilateral regardless of target position. The size of attentional capture was computed for trials in which the target appeared ipsilateral to the TMS site (in the LVF) and contralateral to TMS site (in the RVF) separately (Fig. 4). This analysis showed that the effect of TMS to left parietal cortex affected both visual fields, i.e., the increase in attentional capture was observed both in the LVF [pre-TMS, $t_{(14)}=1.37, p=0.19$; $\operatorname{post}(1), t_{(14)}=2.25, p=0.04$; $\left.\operatorname{post}(2), t_{(14)}=2.47, p=0.02\right]$ and in the RVF [pre-TMS, $t_{(14)}=0.83, p=0.42$; post $(1), t_{(14)}=2.62$, $\left.p=0.02 ; \operatorname{post}(2), t_{(14)}=2.19, p=0.04\right]$. These results suggest that the left SPL serves to suppress distraction from taskirrelevant salient distractors in both visual fields. 

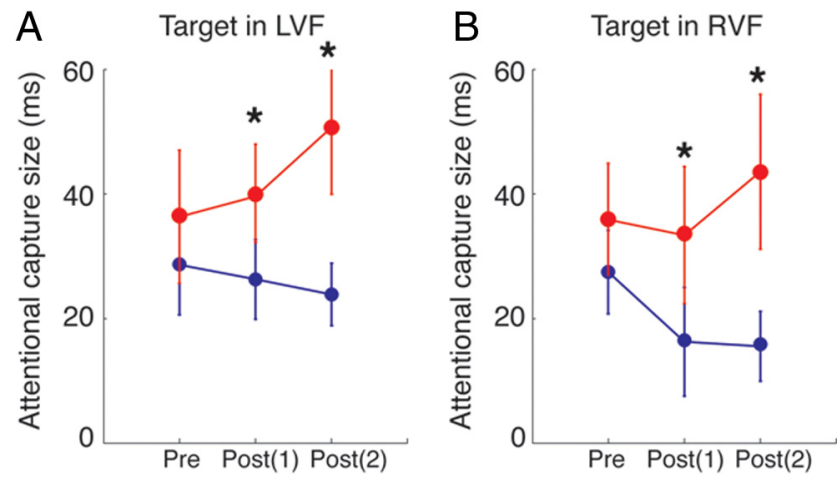

Figure 4. The capture effects before and after theta-burst TMS are plotted for the trials in which the target appeared in the left visual field $(\boldsymbol{A})$ and in the right visual field $(\boldsymbol{B})$. The red circles indicate the parietal TMS condition and the blue squares the control condition. Error bars indicate 1 SEM across 15 participants. The asterisks indicate a significant difference between the conditions $(p<0.05)$.

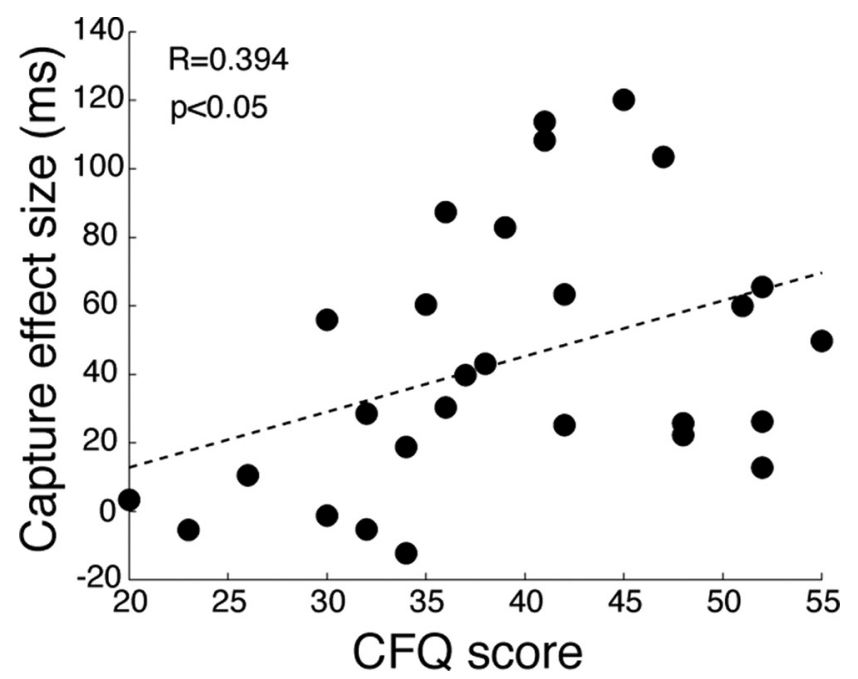

Figure 5. The correlation between CFQ score and attentional capture size (experiment 3; see Materials and Methods) is shown as a scatter plot. Individual participants $(n=28)$ are shown by black dots. The least-squares fit line is indicated by a dashed line.

\section{Correlation between attentional capture and CFQ score}

The TMS experiment supports the hypothesis that the left SPL, whose gray matter volume reflected everyday distractibility as measured by CFQ, plays a causal functional role in suppressing attentional capture. Although the attentional capture task was chosen to quantify distractibility in a laboratory setting, it remains unclear whether attentional capture itself is related to individuals' everyday distractibility. Given the vastly different approaches to measure distractibility of individuals (i.e., a computer-based, abstract visual task involving orientation discrimination vs a pencil-and-paper questionnaire), it is conceivable that the kind of distractibility measured by the two methods may be different. We therefore examined the relationship between the size of attentional capture and the CFQ scores in a total of 28 participants. If these two measures of distractibility share overlapping mechanisms, a systematic relationship should be observed between them.

The scatter plot in Figure 5 depicts the relation between attentional capture size and CFQ scores. As predicted, we found a significant correlation between attentional capture and CFQ score $\left(r=0.394, t_{(26)}=2.18, p<0.05\right)$. Even when we excluded four participants who did not show any attentional capture from the analysis, the positive correlation remained significant $(r=$ $\left.0.344, t_{(22)}=1.72, p<0.05\right)$. These results confirm our intuition that the attentional capture paradigm used in the TMS experiment and self-reported distractibility scores of CFQ test do indeed assess overlapping cognitive and neurobiological phenomena.

\section{Discussion}

We investigated whether interindividual variability in everyday distractibility was reflected in human brain structure. Our VBM results established that variability in regional gray matter density of the left SPL predicted an individual's distractibility in everyday life. The results of a second TMS experiment validated this link by showing a causal relationship between left SPL function and the behavioral effect of attentional distraction caused by taskirrelevant salient stimuli. Specifically, the TMS experiment indicated that the left SPL is involved in the top-down control of attention to avoid distraction.

Our findings that the left SPL is involved in top-down attentional control and suppression of salient task-irrelevant stimuli receives further support from earlier studies. The SPL is activated bilaterally when an irrelevant color singleton is present in a visual search task (de Fockert et al., 2004). Moreover, left parietal cortex is functionally specialized in attentional control (Mevorach et al., 2006, 2009). The left intraparietal sulcus is activated when low saliency stimuli have to be selected while ignoring salient stimuli. In contrast, right parietal cortex responds to saliency per se regardless of task relevance (Mevorach et al., 2009). Furthermore, TMS over the left parietal cortex disrupts the ability to suppress attentional capture by salient stimuli (Mevorach et al., 2006), whereas TMS over the right parietal cortex reduces attentional capture by suppressing the saliency of task-irrelevant stimuli (Hodsoll et al., 2009). These findings converge to the conclusion that the left SPL plays a specific role in suppressing distracting stimuli and offer an explanation for our finding that distractibility was associated with the left SPL, rather than the right SPL.

One previous study has reported a TMS experiment conceptually similar to ours (Hodsoll et al., 2009). In that earlier study, attentional capture was reduced by $1 \mathrm{~Hz}$ repetitive TMS (rTMS) over the right parietal cortex (identified as $\mathrm{P} 4$ in the international 10-20 EEG system), but no significant effect was found with rTMS over the left parietal cortex (P3). The discrepancy between the present study and those earlier findings can be attributed to several factors. First, in the present study we defined the position of left SPL by the MNI coordinate of the cluster found in the VBM study and used MRI neuro-navigation method to position the TMS coil at the location specifically for each individual. In contrast the previous study used the international 10-20 system (i.e., P3 and P4), which leads to differences in statistical power (Sack et al., 2009). Specifically, it is estimated that the international 10-20 system requires $>40$ participants to reach the same statistical power as a TMS study with 14 participants using a coordinatebased targeting with an MRI neuro-navigation system. The number of participants in the present study was 15, whereas it was 8 in the study by Hodsoll et al. (2009). Second, the target site for our left SPL does not correspond to P3 targeted by Hodsoll et al. (2009). When the left SPL was projected to the 10-20 system on the scalp (Olaf Steinstrater, www.uni-muenster.de/ger/t2tconv/ conv3d.html), it corresponded to the midway point between P3 and Pz. This distance on the scalp $(\sim 3-4 \mathrm{~cm})$ is larger than the spatial specificity of TMS, enabling this technique to target separate neuronal populations and produce dissociable results (Pitcher et al., 2009; Kanai et al., 2010, 2011). Thus, it is highly 
likely that the left parietal region stimulated in the study by Hodsoll et al. (2009) was a separate region in the inferior, as opposed to superior, parietal lobe. Third, the TMS protocols were different between the two studies: we used 40 s cTBS, whereas Hodsoll et al. (2009) used $1 \mathrm{~Hz}$ rTMS for $10 \mathrm{~min}$. All of these methodological differences could contribute to the differences in the results.

Our results could be interpreted in terms of a criterion shift in the decision threshold. The activity of neuronal populations in parietal cortex correlates with perceptual decisions in nonhuman primates (Shadlen and Newsome, 1996, 2001; Hanks et al., 2006; Kiani and Shadlen, 2009) and human subjects (Heekeren et al., 2004; Ploran et al., 2007; Tosoni et al., 2008; Ho et al., 2009). If the activity in the left SPL were important for keeping the response decision threshold low, disruption of this region would make the decision threshold higher, thus delaying response times. This idea is compatible with the increase in reaction times that we observed following TMS over the left SPL (Fig. 3A,B). However, this account would also predict a speed-accuracy trade-off (Bogacz et al., 2010), and a more conservative criterion would also result in higher accuracy following TMS. However, we did not observe any effect of TMS on accuracy in the present study (Fig. 3C,D). Since the accuracy was generally very high $(\sim 90 \%)$ in our experiment (Fig. $3 C, D$ ), the absence of a TMS effect on accuracy may simply reflect a ceiling effect. It will therefore be interesting for future work to test the possibility that the left SPL maintains a low threshold for decision using a task (e.g., random dot motion task) suitable for teasing apart elements that affect speed and accuracy in decision making (Gold and Shadlen, 2007).

We found that the gray matter density of the left SPL was larger among highly distractible individuals. This positive correlation is interesting, because it has previously been implicitly assumed that a larger cortical volume or greater gray matter density is associated with better performance. Here, we show that greater gray matter density in adults can be associated with poorer performance. A number of possible explanations can be offered from a developmental perspective.

During adolescence the synapses in human cortex undergo a pruning process (Huttenlocher and Dabholkar, 1997), which is supposedly linked with improved processing efficacy of pruned cortical regions. As the reduction of gray matter density due to the pruning process occurs between childhood and early adult life across broad areas of the cortex, including the left SPL, more mature brains tend to have smaller SPL (Gogtay et al., 2004). Considering cortical pruning as a process of maturation, it is conceivable that smaller volume (or gray matter density) of the left SPL leads to more efficient attentional control by this region. In fact, situations where smaller cortical volumes are associated with better behavioral performance have been reported in music perception (Hyde et al., 2007), in autism (Hyde et al., 2010), and in the ability to manipulate self-generated thoughts (Dumontheil et al., 2010). While this maturation hypothesis can provide an explanation as to how smaller gray matter volume in a cortical area could lead to better behavioral performance, further studies are needed to determine when larger cortex leads to poorer performance.

An alternative explanation for our findings is that in highly distractible individuals the left SPL has undergone compensatory structural changes to provide them with essential top-down control to function normally in daily life (the compensation hypothesis). However, this interpretation assumes that the actual source of distractibility is elsewhere and the left SPL only plays a compensatory role. While this interpretation is compatible with the TMS results, importantly, our VBM analysis did not reveal any region that showed a stronger correlation with the distractibility score than the left SPL (or indeed any other significant correlations). The only area that showed negative correlation was the left prefrontal cortex, but the correlation was weak and did not reach a statistically significant level (Fig. $1 B$ ). If this area were the primary cause of distractibility in everyday life, it would be expected to show a more direct link with the distractibility scores than secondary areas showing compensatory growth in response to the deficiency. Note that both the maturation hypothesis and compensation hypothesis assume that the role of the left SPL is to control attention in the presence of distracting stimuli and are both compatible with the TMS results.

A recent study showed that chronic multitaskers in the media environment are worse at filtering out interference from irrelevant information (Ophir et al., 2009). Our results predict that heavy media multitaskers who are highly susceptible to distraction are likely to have greater gray matter density in the left SPL. However, the causal relationship between distractibility and multitasking in everyday life remains to be resolved, and it will be interesting to examine how our media-rich online culture influences individual brain structures and distractibility.

In summary, we have shown that interindividual variability in self-report measures of distractibility in everyday life are predicted by the focal gray matter density in left superior parietal cortex. Using TMS, we have causally linked the function of this left superior parietal region to maintaining top-down control in the presence of task-irrelevant distraction.

\section{References}

Ashburner J (2007) A fast diffeomorphic image registration algorithm. Neuroimage 38:95-113.

Bogacz R, Wagenmakers EJ, Forstmann BU, Nieuwenhuis S (2010) The neural basis of the speed-accuracy tradeoff. Trends Neurosci 33:10-16.

Boomsma DI (1998) Genetic analysis of cognitive failures (CFQ): a study of Dutch adolescent twin pairs and their parents. Eur J Pers 12:321-330.

Broadbent DE, Cooper PF, FitzGerald P, Parkes KR (1982) The cognitive failures questionnaire (CFQ) and its correlates. Br J Clin Psychol 21:1-16.

Collins DL, Holmes CJ, Peters TM, Evans AC (1995) Automatic 3-D modelbased neuroanatomical segmentation. Hum Brain Mapp 3:190-208.

Corbetta M, Shulman GL (2002) Control of goal-directed and stimulusdriven attention in the brain. Nat Rev Neurosci 3:201-215.

Davies DR, Jones DM, Taylor A (1984) Individual differences in attention. In: Varieties of attention (Parasuraman R, Davies DR, eds), pp 395-447. New York: Academic.

de Fockert J, Rees G, Frith C, Lavie N (2004) Neural correlates of attentional capture in visual search. J Cogn Neurosci 16:751-759.

Desimone R, Duncan J (1995) Neural mechanisms of selective visual attention. Annu Rev Neurosci 18:193-222.

Dumontheil I, Hassan B, Gilbert SJ, Blakemore S-J (2010) Development of the selection and manipulation of self-generated thoughts in adolescence. J Neurosci 30:7664-7671.

Forster S, Lavie N (2007) High perceptual load makes everybody equal. Psychol Sci 18:377-381.

Gogtay N, Giedd JN, Lusk L, Hayashi KM, Greenstein D, Vaituzis AC, Nugent TF 3rd, Herman DH, Clasen LS, Toga AW, Rapoport JL, Thompson PM (2004) Dynamic mapping of human cortical development during childhood through early adulthood. Proc Natl Acad Sci U S A 101:8174-8179.

Gold JI, Shadlen MN (2007) The neural basis of decision making. Annu Rev Neurosci 30:535-574.

Hanks TD, Ditterich J, Shadlen MN (2006) Microstimulation of macaque area LIP affects decision-making in a motion discrimination task. Nat Neurosci 9:682-689.

Heekeren HR, Marrett S, Bandettini PA, Ungerleider LG (2004) A general mechanism for perceptual decision-making in the human brain. Nature 431:859-862.

Ho TC, Brown S, Serences JT (2009) Domain general mechanisms of perceptual decision making in human cortex. J Neurosci 29:8675-8687.

Hodsoll J, Mevorach C, Humphreys GW (2009) Driven to less distraction: 
rTMS of the right parietal cortex reduces attentional capture in visual search. Cereb Cortex 19:106-114.

Huang Y-Z, Edwards MJ, Rounis E, Bhatia KP, Rothwell JC (2005) Theta burst stimulation of the human motor cortex. Neuron 45:201-206.

Huttenlocher PR, Dabholkar AS (1997) Regional differences in synaptogenesis in human cerebral cortex. J Comp Neurol 387:167-178.

Hyde KL, Lerch JP, Zatorre RJ, Griffiths TD, Evans AC, Peretz I (2007) Cortical thickness in congenital amusia: when less is better than more. J Neurosci 27:13028-13032.

Hyde KL, Samson F, Evans AC, Mottron L (2010) Neuroanatomical differences in brain areas implicated in perceptual and other core features of autism revealed by cortical thickness analysis and voxel-based morphometry. Hum Brain Mapp 31:556-566.

Itti L, Koch C (2001) Computational modeling of visual attention. Nat Rev Neurosci 2:194-203.

Kanai R, Bahrami B, Rees G (2010) Human parietal cortex structure predicts individual differences in perceptual rivalry. Curr Biol 20:1626-1630.

Kanai R, Carmel D, Bahrami B, Rees G (2011) Structural and functional fractionation of right superior parietal cortex in bistable perception. Curr Biol 21:R106-R107.

Kastner S, Ungerleider LG (2000) Mechanisms of visual attention in the human cortex. Annu Rev Neurosci 23:315-341.

Kiani R, Shadlen MN (2009) Representation of confidence associated with a decision by neurons in the parietal cortex. Science 324:759-764.

Larson GE, Merritt CR (1991) Can accidents be predicted? An empirical test of the Cognitive Failures Questionnaire. Appl Psychol 40:37-45.

Martin M, Jones GV (1983) Distribution of attention in cognitive failure. Hum Learn 2:221-226.

Mazziotta J, Toga A, Evans A, Fox P, Lancaster J, Zilles K, Woods R, Paus T, Simpson G, Pike B, Holmes C, Collins L, Thompson P, MacDonald D, Iacoboni M, Schormann T, Amunts K, Palomero-Gallagher N, Geyer S, Parsons L, et al. (2001) A probabilistic atlas and reference system for the human brain: international consortium for brain mapping (ICBM). Philos Trans R Soc Lond B Biol Sci 356:1293-1322.

Mevorach C, Humphreys GW, Shalev L (2006) Opposite biases in saliencebased selection for the left and right posterior parietal cortex. Nat Neurosci 9:740-742.

Mevorach C, Shalev L, Allen HA, Humphreys GW (2009) The left intraparietal sulcus modulates the selection of low salient stimuli. J Cogn Neurosci 21:303-315.
Ophir E, Nass C, Wagner AD (2009) Cognitive control in media multitaskers. Proc Natl Acad Sci U S A 106:15583-15587.

Pitcher D, Charles L, Devlin JT, Walsh V, Duchaine B (2009) Triple dissociation of faces, bodies and objects in extrastriate cortex. Curr Biol 19:319-324.

Ploran EJ, Nelson SM, Velanova K, Donaldson DI, Petersen SE, Wheeler ME (2007) Evidence accumulation and the moment of recognition: dissociating perceptual recognition processes using fMRI. J Neurosci 27:11912-11924.

Sack AT, Cohen Kadosh R, Schuhmann T, Moerel M, Walsh V, Goebel R (2009) Optimizing functional accuracy of TMS in cognitive studies: a comparison of methods. J Cogn Neurosci 21:207-221.

Shadlen MN, Newsome WT (1996) Motion perception: seeing and deciding. Proc Natl Acad Sci U S A 93:628-633.

Shadlen MN, Newsome WT (2001) Neural basis of a perceptual decision in the parietal cortex (area LIP) of the rhesus monkey. J Neurophysiol 86:1916-1936.

Theeuwes J (1991) Exogenous and endogenous control of attention: the effect of visual onsets and offsets. Percept Psychophys 49:83-90.

Theeuwes J (1992) Perceptual selectivity for color and form. Percept Psychophys 51:599-606.

Theeuwes J (1994) Endogenous and exogenous control of visual selection. Perception 23:429-440.

Tipper SP, Baylis GC (1987) Individual differences in selective attention: the relation of priming and interference to cognitive failure. Pers Individ Diff 8:667-675.

Tosoni A, Galati G, Romani GL, Corbetta M (2008) Sensory-motor mechanisms in human parietal cortex underlie arbitrary visual decisions. Nat Neurosci 11:1446-1453.

Wallace JC (2004) Confirmatory factor analysis of the cognitive failures questionnaire: evidence for dimensionality and construct validity. Pers Individ Diff 37:307-324.

Wallace JC, Vodanovich SJ (2003) Can accidents and industrial mishaps be predicted? Further investigation into the relationship between cognitive failure and reports of accidents. J Bus Psychol 17:503-514.

Wallace JC, Kass SJ, Stanny CJ (2002) Cognitive failures questionnaire revisited: correlates and dimensions. J Gen Psychol 129:238-256.

Yantis S, Jonides J (1984) Abrupt visual onsets and selective attention: evidence from visual search. J Exp Psychol Hum Percept Perform 10:601-621.

Yantis S, Jonides J (1990) Abrupt visual onsets and selective attention: voluntary versus automatic allocation. J Exp Psychol Hum Percept Perform $16: 121-134$. 